\title{
Correlación entre elastografía tiroidea y anatomía patológica de nódulos tiroideos
}

\section{Correlation between thyroid elastography and pathological anatomy of thyroid nodules}

\author{
María Florencia Elías Kairuz (D), Diego Riolo. \\ 1 Universidad Católica de Córdoba, Facultad de Ciencias de la Salud, Instituto Oulton, Servicio de Ecografía General. \\ Correspondencia: María Florencia Elías Kairuz, e-mail: floreliask@ hotmail.com.
}

\section{Resumen}

INTRODUCCION: Frente a la alta prevalencia de enfermedad y nódulos tiroideos en nuestra población (Argentina), en el ámbito de la práctica clínica, lo importante ante un nódulo tiroideo es determinar su naturaleza benigna o maligna, puesto que su manejo clínico es muy diferente.

Tras su detección clínica, se suele practicar una ecografía, la cual tiene un papel fundamental para el diagnóstico, poniendo en evidencia signos ecográficos indicadores de benignidad o malignidad. Por esto, se desarrolló el sistema TI-RADS, para clasificar la probabilidad de malignidad/benignidad de los nódulos tiroideos en imagen ecográfica el modo B y Doppler color.

Sin embargo, estos criterios ecográficos no tienen suficiente sensibilidad y especificidad, por lo que en muchos casos se recurre al examen citológico, el Gold stándar, para confirmar el diagnóstico. La biopsia con punción con aguja fina bajo control ecográfico es un procedimiento mínimamente invasivo, pero no exento de riesgos ni resultados falsos negativos. Por esto la introducción de la elastografía para el estudio de los nódulos tiroideos se propuso con el objetivo de incrementar el rendimiento diagnóstico de la ecografía, y así poder elegir más criteriosamente cuál nódulo merece un estudio citológico.

La elastografía comprende un conjunto de técnicas que permiten la elasticidad de los tejidos. La dureza y los valores de elasticidad obtenidos estarán en relación directa a la severidad de la patología: a mayor afectación histológica, mayor rigidez y por lo tanto mayor probabilidad de malignidad.

OBJETIVO: Analizar la capacidad diagnóstica de la elastografía, para diferencias nódulos tiroides benignos y malignos, utilizando como prueba de referencia el análisis anatomopatologico de la citología obtenida por punción con aguja fina bajo control ecográfico.

MATERIALES Y METODOS: Estudio observacional descriptivo y retrospectivo.

Pacientes de ambos sexos, mayores de edad, que asistieron al servicio con indicación de PAAF guiada por ecografía, de nódulo tiroideo, previamente diagnosticado $(n=81)$, en un periodo comprendido entre los meses de abril del año 2019 al mes de enero del año 2020, inclusive. A todos se les realizó previo a la punción, elastografía shear wave, obteniendo valores en $\mathrm{kPa}$. Se utilizó como valor de corte $65 \mathrm{kPa}$ (valores menores indicarían benignidad, y valores mayores malignidad).

RESULTADOS: Se realizó elastografía tiroidea a 81 pacientes con nódulo tiroideo previamente diagnosticado, obteniendo 56 resultados benignos, 21 malignos y 4 bordeline. En el 82,7\% de los nódulos estudiados hubo correlación con el resultado obtenido por elastografía con el anatomopatologico, tanto benigno como maligno.

CONCLUSIÓN: La probabilidad de malignidad de un nódulo tiroideo, según este estudio, está directamente relacionada con los valores de la elastografía. Por consiguiente, la elastografía SWE permite

Revista Methodo: Investigación Aplicada a las Ciencias Biológicas. Universidad Católica de Córdoba. Jacinto Ríos 571 Bo Gral. Paz. X5004FXS. Córdoba. Argentina. Tel.: (54) 3514517299 / Correo: methodo@ucc.edu.ar /Web: methodo.ucc.edu.ar | ARTICULO ORIGINAL Rev. Methodo 2022;7(1):13-23. 
predecir la benignidad de un nódulo y consecuentemente reducir el número de punciones aspirativas con aguja fina.

Palabras claves: nódulos tiroideos, elastografía tiroidea, TI-RADS, cáncer de tiroides.

\section{Abstract}

INTRODUCTION: Faced with the high prevalence of thyroid disease and nodules in our population (Argentina), in the field of clinical practice, the important thing when dealing with a thyroid nodule is to determine its benign or malignant nature, since its clinical management is very different.

After its clinical detection, an ultrasound is usually performed, which has a fundamental role for the diagnosis, showing ultrasound signs that indicate benignity or malignancy. For this reason, the TI-RADS system was developed to classify the probability of malignancy / benignity of thyroid nodules in B-mode ultrasound imaging and Doppler color.

However, these ultrasound criteria don't have sufficient sensitivity and specificity, so that in many cases a cytological examination, the gold standard, is used to confirm the diagnosis. Fine needle biopsy under ultrasound control is a minimally invasive procedure, but it is not without risks or false negative results. For this reason, the introduction of elastography for the study of thyroid nodules was proposed with the aim of increasing the diagnostic performance of ultrasound, and thus being able to choose more carefully which nodule deserves a cytological study.

Elastography comprises a set of techniques that allow the elasticity of tissues. The hardness and elasticity values obtained will be directly related to the severity of the pathology: the greater the histological involvement, the greater the rigidity and therefore the greater the probability of malignancy.

OBJECTIVE: To analyze the diagnostic capacity of elastography, for differences in benign and malignant thyroid nodules, using the pathological analysis of the cytology obtained by FNA as a reference test.

MATERIALS AND METHODS: Descriptive and retrospective observational study.

Patients of both sexes, of legal age, who attended the Service with an indication for fine needle biopsy under ultrasound control guided by ultrasound, of a previously diagnosed thyroid nodule $(\mathrm{n}=81)$, in a period between the months of April 2019, to the month of January of the year 2020 inclusive. All of them underwent SWE elastography (shear wave elastography) prior to puncture, obtaining values in $\mathrm{kPa}$. $65 \mathrm{kPa}$ was used as cut-off value (lower values would indicate benignity, and higher values would indicate malignancy).

RESULTS: Thyroid elastography was performed in 81 patients with a previously diagnosed thyroid nodule, obtaining 56 benign results, 21 malignant and 4 borderline. In $82.7 \%$ of the nodules studied, there was a correlation between the result obtained by elastography and the pathological one, both benign and malignant.

CONCLUSION: The probability of malignancy of a thyroid nodule, according to this study, is directly related to the elastography values. Consequently, SWE elastography makes it possible to predict the benignity of a nodule and consequently reduce the number of fine needle aspiration punctures.

Keywords: thyroid nodule, thyroid elastography, TI-RADS, thyroid cancer.

\section{Introducción}

Los nódulos tiroideos son altamente prevalentes en la población adulta (del 3,7 \% al 4,5\%), predominantemente en mujeres y en áreas yodosuficientes $^{1-2}$. En nuestra población (Córdoba, Argentina) se detectó mediante la palpación tiroidea, la presencia de nódulos tiroideos en un $13 \%$ de los pacientes examinados en una campaña realizada en el año $2015^{3}$. Un tercio de los adultos presentan uno o más nódulos durante la exploración ecográfica ${ }^{4}$.

Frente a esta prevalencia, la enfermedad nodular tiroidea supone un dilema diagnóstico para el especialista, al tratar de diferenciar cuáles de estos nódulos son malignos. Los hallazgos post quirúrgicos han revelado qué 5-15\% de todos los nódulos enviados a cirugía resultan cáncer ${ }^{5-6}$. Factores como: edad, sexo, exposición a la radiación, historia familiar, inducen a sospechar malignidad ante la presencia de un nódulo tiroideo, sin embargo, no es claro si existen otros parámetros que puedan ser predictores de malignidad.

Los adenomas consisten en una lesión encapsulada, derivada del epitelio folicular; pueden ser funcionantes o no funcionantes. Los adenomas funcionantes (autónomos) son 
proporcional-mente más grandes que el resto del parénquima y producen una cantidad excesiva de hormonas tiroideas; en los adenomas no funcionantes, los niveles de hormonas tiroideas no se ven alteradas. Estos nódulos son considerados generalmente benignos, y rara vez malignizan. Los nódulos hiperplásicos se presentan con mayor frecuencia en el bocio multinodular y son causados por la hiperplasia de las células foliculares. Los carcinomas diferenciados de tiroides (carcinoma papilar y folicular), son tumores poco frecuentes, entre 1-10/100.000 casos por año. Son la neoplasia endocrina más prevalente en la población, tienen buen pronóstico y re-presentan solo el $1 \%$ de todas las lesiones tumorales malignas. Los carcinomas indiferenciados $\mathrm{o}$ anaplásicos representan el 5\% de los tumores de tiroides, y el carcinoma medular (derivado de las células para-foliculares) se presenta esporádicamente o es de aparición familiar.

La ecografía de alta resolución es el método de imágenes de primera línea para detectar lesiones nodulares en la glándula. Los ecógrafos de gran resolución espacial con que contamos en la actualidad, sumado a la localización superficial de la glándula en el cuello, hacen posible la detección de nódulos milimétricos. Además, es un método de imagen no invasivo, de bajo costo, de amplia disponibilidad y no utiliza radiación ionizante.

La PAAF es el mejor método diagnóstico no quirúrgico para detectar malignidad, sin embargo, es un procedimiento invasivo, sujeto a errores en la toma de la muestra, consume recursos económicos y tiempo, y presenta pocas complicaciones y de escasa gravedad.

Es importante definir qué nódulos requieren biopsia por aspiración con aguja fina (PAAF) para disminuir los riesgos de un procedimiento innecesario y evitar que pase inadvertido el diagnóstico de neoplasia maligna.

A lo largo de un sinfín de investigaciones que tuvieron como propósito caracterizar los nódulos tiroideos y los criterios que ayudarían a determinar qué pacientes necesitaban un estudio citológico y cuándo un procedimiento invasivo resulta innecesario y podría ser evitado, se propusieron distintos criterios de ultrasonografía para establecer el riesgo de malignidad de los nódulos tiroideos. La mayoría de los autores los dividen en nódulos benignos (coloides), lesiones foliculares, y nódulos malignos. La dificultad estriba, en que, estos estudios general-mente no son rigurosos desde el punto de vista metodológico y no evalúan de la misma manera las distintas variables ecográficas. Sumado a ello el equipo empleado no es uniforme, utilizándose en algunos estudios transductores de $7 \mathrm{MHz}$ y en otros de mayores frecuencias (12-14 MHz). El resultado es la gran variabilidad de conclusiones en los estudios disponibles.

La American College of Radiology desarrollo el sistema BI-RADS (Breast Imaging Reporting and Data System) para clasificar las lesiones en glándula mamaria. En base a éste se planteó el homólogo para lesiones tiroideas, TI-RADS (Thyroid Imaging Reporting Data System) propuesto por Horvath et al en el año 2009 (Tabla 1). En la actualidad éste es ampliamente aplicado a la práctica médica diaria en muchos países ${ }^{7}$.

Tabla 1. Clasificación ACR TI-RADS.

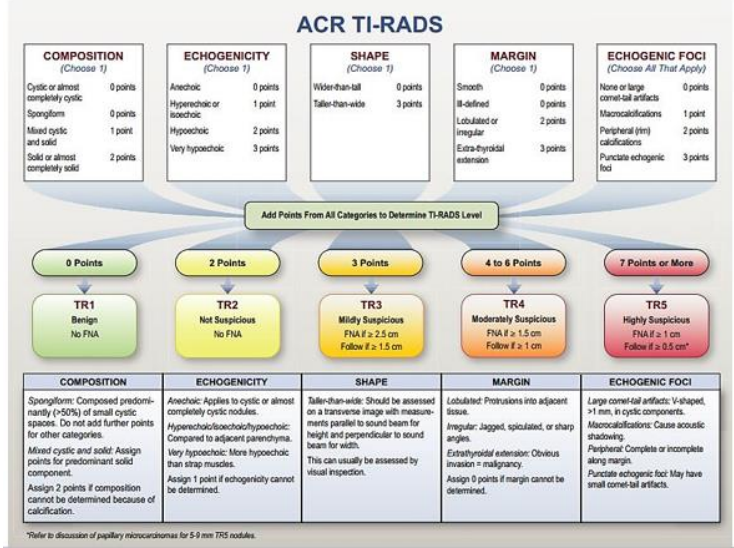

La clasificación de ACR TI-RADS considera las siguientes variables: ecoestructura, ecogenicidad, forma, orientación, bordes, superficie, presencia o ausencia de cápsula, calcificaciones y vascularización del nódulo. Estableciendo así, cinco categorías expuestas también en la (Tabla 1)

TIRADS 1: Nódulo benigno (espongiformes o quistes). Recomendación: seguimiento ecográfico, no PAAF.

TIRADS 2: Nódulo no sospechosos. Recomendación: seguimiento ecográfico, no PAAF.

TIRADS 3: Nódulo probablemente benigno. Recomendación: seguimiento ecográfico si mide más de $1,5 \mathrm{~cm}$ o PAAF si mide más de $2,5 \mathrm{~cm}$.

TIRADS 4: Nódulo moderadamente sospechoso. Recomendación: seguimiento ecográfico si mide más de $1 \mathrm{~cm}$ o PAAF si mide más de $1,5 \mathrm{~cm}$.

TIRADS 5: Nódulo altamente sospechoso. Recomendación: PAAF.

A diferencia de otros sistemas de categorización, ACR-TIRADS:

-No incluye subcategorías.

- No integra patrones morfológicos definidos, tiroiditis Hashimoto, White knight, etc.

- No incluye una categoría TR0 para indicar una glándula tiroides normal.

- No incluye vascularización tiroidea como signo o no de sospecha.

-No incluye patrón de elastografía. 
-Menciona criterios de sospecha en ganglios, pero no lo incluye en la clasificación.

Según estudios e investigaciones previas donde se han comparado la clasificación TIRADS con resultados anatomopatológicos, la conclusión general indica que nódulos categorizados como TIRADS 3, 4 y 5, según el tamaño, deberían ser estudiados con PAAF. Si bien esta clasificación es de fácil aplicación y muy útil en el diagnóstico de nódulos tiroideos, ofrece varias limitaciones, la principal es el componente subjetivo de que es observador dependiente.

A lo largo de la historia los informes de PAAF fueron descriptivos, no sistematizados, no comparables y rara vez se indicaba el manejo del paciente ${ }^{8}$.

Esto ha cambiado radicalmente después de la reunión multidisciplinar de expertos en patología tiroidea celebrada en Bethesda 2007 (Tabla 2), donde se establece el manual de la nomenclatura y Sistemática de elaboración de los informes de PAAF tiroidea. Dicha clasificación establece seis categorías diagnósticas e indica en cada una de ellas el manejo clínico-terapéutico del paciente.

Tabla 2. Clasificación Bethesda.

\begin{tabular}{|l|l|l|}
\hline Categoria diagnóstica & Riesgo de malignidad (\%) & Manejo usual \\
\hline $\begin{array}{l}\text { I No diagnóstico o insatis- } \\
\text { factorio }\end{array}$ & depende & $\begin{array}{l}\text { Repetir biopsia con mono- } \\
\text { grafia }\end{array}$ \\
\hline II Benigno & $0-3$ & Seguimiento clínico \\
\hline $\begin{array}{l}\text { III Atipia de significado in- } \\
\text { determinado o lesión folicu- } \\
\text { lar indeterminada }\end{array}$ & $\begin{array}{l}\text { Repetir biopsia con sonogra- } \\
\text { fia }\end{array}$ \\
\hline $\begin{array}{l}\text { IV Neoplasia folicular } \\
\text { (NF), sospecha de NF }\end{array}$ & $15-30$ & Lobectomía \\
\hline V Sospecha de malignidad & $60-75$ & $\begin{array}{l}\text { Lobectomía o tiroidectomía } \\
\text { total }\end{array}$ \\
\hline VI Maligno & $97-99$ & Tiroidectomía total \\
\hline
\end{tabular}

Recientemente se ha desarrollado un nuevo modo de imagen no invasivo llamado elastografía, el cual comprende un conjunto de técnicas de diagnóstico que utiliza el ultrasonido y permiten estudiar una de las propiedades físicas de los tejidos: la viscoelasticidad, aportando información sobre la rigidez o elasticidad del tejido, midiendo el nivel de distorsión sufrido por estos bajo la aplicación de una fuerza externa y así estudiar la organización estructural del mismo.

La rigidez de los tejidos se puede definir por su módulo de elasticidad: cuanto más rígido y resistente a la deformación, menos elástico es el tejido ${ }^{9}$.

Existen tres tipos de elasticidad

-Elasticidad longitudinal (módulo de Young): es la resistencia al estrés longitudinal. Expresa el cambio de longitud de un tejido al ser sometido a una tensión de compresión o de tracción.

-Elasticidad transversal o de cizallamiento (shear): expresa el cambio de forma de un tejido sometido a una tensión de cizallamiento, con el objetivo de causar deslizamiento de los planos paralelos.

-Elasticidad de volumen (módulo de Bulk): cambio de volumen de un material sometido a una fuerza perpendicular a su superficie ${ }^{10}$.

Los tejidos sólidos resisten los cambios de forma y volumen, por eso poseen rigidez o elasticidad de torsión y elasticidad de volumen. Los líquidos resisten un cambio de volumen, pero no de forma, por eso solo poseen elasticidad de volumen.

Las ondas mecánicas en los cuerpos sólidos pueden ser de dos tipos

Ondas longitudinales: el movimiento de las partículas en el sólido es paralelo a la dirección de propagación de las ondas. La velocidad de estas ondas es de unos $1.540 \mathrm{~m} / \mathrm{s}$, las cuales son los ultrasonidos y son las usadas en ecografía. (Figura 1A)

-Ondas transversales (share waves): el movimiento de las partículas se mueve de arriba hacia abajo mientras la onda se propaga en forma horizontal. Genera ondas de bajas frecuencias de $10 \mathrm{~Hz}-2000$ $\mathrm{Hz}$ con una velocidad de propagación de 1-10 m/s (Figura 1B). Esta baja velocidad permite grandes diferencias entre los tejidos, lo que se traduce como contraste tisular que es detectado mediante la elastografía ${ }^{11}$.
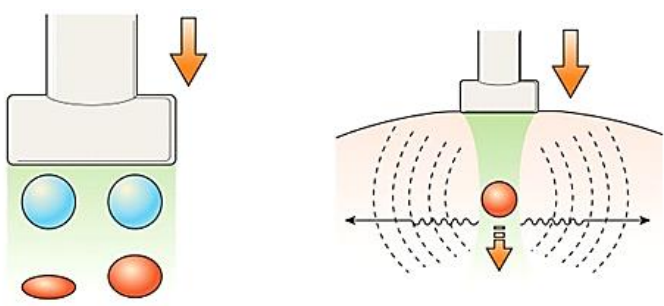

A

Figura 1. Principio de la elastografía. Ondas longitudinales (A), paralelaras a la dirección de la onda del transductor, y ondas transversales, con dirección perpendicular (B).

Estas ondas requieren un medio elástico para su propagación, de tal manera que en los fluidos no pueden utilizarse, a excepción de los fluidos muy viscosos, que se comportan como tejidos blandos. Por lo tanto, cuanta mayor sea la rigidez que tiene el medio, mayor será la velocidad de propagación de las ondas.

Como las ondas transversales se desplazan a una velocidad muy baja, se necesitan equipos rápidos para generar imágenes a unas tasas elevadas, de 6000-20000 imágenes por segundo. Se utilizan 
equipos de ultrasonido convencional al que se le añade el software específico.

Las técnicas elastográficas se basan en la aplicación de esfuerzos externos o internos, y en el análisis de la respuesta mecánica del tejido a dichos esfuerzos.

Aunque a lo largo del tiempo se han ido desarrollando diversas formas de elastografía, las dos modalidades que hoy en día se emplean con más frecuencia en la práctica clínica son la strain elastography (SE, elastografía estática) y la shear wave elastography (SWE, elastografía dinámica).

\section{Elastografía de Strain}

Para evaluar la relación entre compresión y deformación, se ha utilizado el módulo de Young, la relación de presión y deformación. La elastografía Strain (o estática) requiere una estimulación externa con una sonda o estrés endógeno, como movimientos cardiovasculares, que resultan en un desplazamiento axial del tejido por estrés mecánico.

La deformación tisular del estrés se mide y visualiza en un modo de pantalla dividida con una imagen convencional en modo B y un elastograma. Para adquirir las imágenes elastográficas, la compresión se aplica continuamente mediante un transductor y se sigue por una descompresión (Figura 1A). La imagen elástica se superpone al modo B y se muestran en un continuo de colores de rojo (tejido blando) a azul (tejido duro), de acuerdo a lo predefinido, las escalas de color se pueden aplicar inversamente (Figura 2).

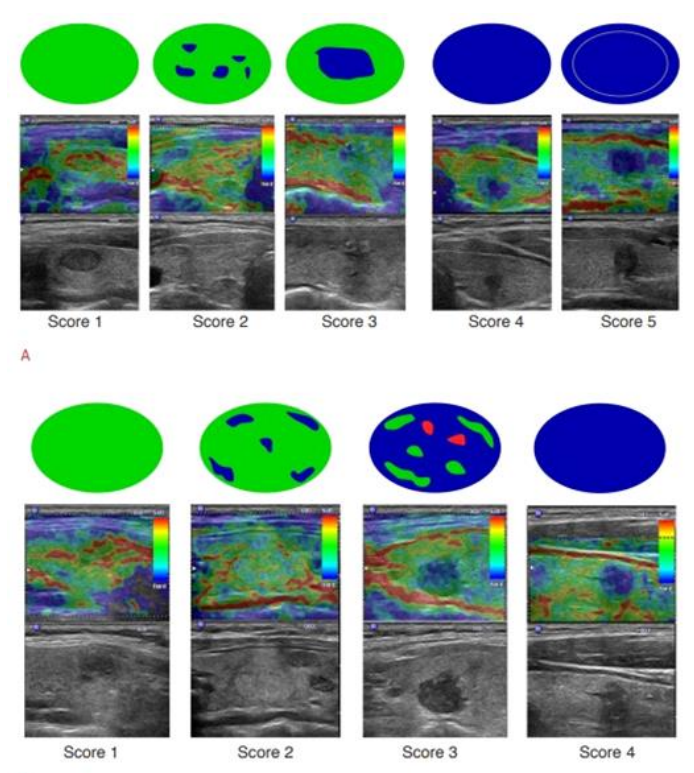

Figura 2. Evaluación cualitativa de la elastografía Strain.

Por elastografía de Strain, se pueden obtener la evaluación de elasticidad dibujando dos regiones de interés (ROIs) sobre región objetivo y la región de referencia adyacente. Luego, una relación de deformación se calcula automáticamente a través del sistema.

La probabilidad de malignidad aumenta con un aumento en la relación.

\section{Elastografía Shear Wave}

Las Shear Wave u ondas de corte, son los componentes transversales del desplazamiento de partículas que son rápidamente atenuados por el tejido (Figura 1B). Su velocidad está estrechamente relacionada con la fórmula del módulo de Young, en la cual la elasticidad del tejido puede ser evaluado a partir de la velocidad de propagación de la onda de corte.

La elastografía Shear Wave (SWE) proporciona información elástica cuantitativa sobre la base del pulso acústico de una sonda de ultrasonido que estimula los tejidos, produciendo un elastograma en tiempo real. Cómo SWE depende de la producción de fuerza producida por la sonda, es más independiente del operador, reproducible y cuantitativa absoluta.

Hay dos métodos aplicables para la evaluación de nódulos tiroideos: la onda de corte supersónica (ESS) y fuerza de impulso de radiación acústica (ARFI).

El primero usa ultrasonidos enfocados que se propagan a través de toda el área de imágenes. Un código de imagen muestra la velocidad de la onda de corte $(\mathrm{m} / \mathrm{seg})$ o elasticidad (kilopascales, $\mathrm{kPa}$ ) para cada píxel en el ROI. Dentro de un ROI, se puede medir una variedad de parámetros de rigidez, que incluyen la rigidez media (Emean), rigidez máxima (Emax) y desviación estándar (SD). A diferencia de la elastografía Strain, se muestra el tejido blando en tonos de azul y el tejido duro se muestra en rojo (Figura 3 ).

ARFI usa pulsos acústicos de corta duración que excitan el tejido dentro del ROI. La elasticidad se expresa en metros por segundo $(\mathrm{m} / \mathrm{seg}$ ) y no muestra imágenes codificadas por colores para la elastografía. Determinadas patologías modifican los parámetros visco elásticos de los tejidos blandos. En los tejidos biológicos, la elasticidad se ve influida por algunos elementos como la grasa tisular y el grado de fibrosis. Los tejidos blandos son viscoelásticos, de tal manera que la recuperación de la forma y tamaño tras la deformación no es inmediata, además de que la elasticidad varía dependiendo de la dirección y del grado de deformación.

En el estudio de nódulos tiroideos, la elastografía podría ser de gran utilidad si se utiliza junto con la ecografía convencional, permitiendo excluir 
malignidad, con mayor probabilidad, si el estudio elastográfico es negativo.

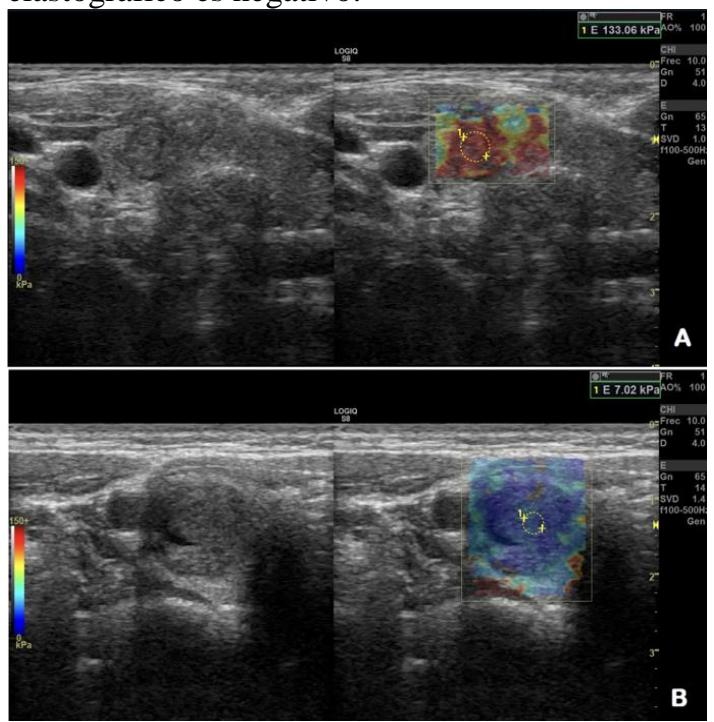

Figura 3. Evaluación cuantitativa de la elastografía Share Wave. La elasticidad del nódulo se expresa en la unidad de kilopascales $(\mathrm{kPa})$ en el Q-Box dentro del ROI.

La elastografía también se ha aplicado en la valoración de algunas enfermedades tiroideas difusas y procesos inflamatorios autoinmunes, como la tiroiditis crónica autoinmune de Hashimoto. En ellas se ha detectado alteración de la elasticidad tisular con incrementos en la misma que podrían superponerse a los valores propios del tejido tumoral maligno. Por ello, algunos consideran que la elastografía no es útil para diferenciar entre estas patologías y el cáncer de tiroides, ni tras haber recibido tratamiento quirúrgico o con radio yodó. Tampoco está demostrada su capacidad en el carcinoma folicular y en nódulos quísticos ni calcificados.

Los valores de corte para SWE medidos para las diversas lesiones tiroideas son:

Tiroides normal

ARFI, $2 \pm 0.40 \mathrm{~m} / \mathrm{s}^{12}$

EES, $20.8 \pm 10.4 \mathrm{kPa}^{13}$

Nódulos benignos

ARFI, $<2.34 \pm 1.17 \mathrm{~m} / \mathrm{s}^{12,14-16}$

EES, $<65 \mathrm{kPa}^{13,17}$

Nódulos malignos

ARFI, $\geq 2.34 \pm 1.17 \mathrm{~m} / \mathrm{s}^{12,14-16}$

$\mathrm{EES}, \geq 65 \mathrm{kPa}^{13,17}$
Enfermedad tiroidea difusa

ARFI, $2.07 \pm 0.44 \mathrm{~m} / \mathrm{s}^{18-19}$

EES, $24.0 \pm 10.5 \mathrm{kPa}^{20}$

\section{Objetivo}

Demostrar si la realización de la elastografía shearwave cuantitativa en nódulos tiroideos contribuye a caracterizar las lesiones, aumentando su especificidad y sensibilidad (pues-to que niveles más altos de rigidez se asocian a procesos patológicos neoplásicos malignos, correlacionando con resultados anátomopatológicos realizados en nuestro centro, utilizando como valor de corte $65 \mathrm{kPa}$ para nódulos tiroideos.

\section{Objetivos secundarios}

Establecer en base a los hallazgos ecográficos si existe correlación de estos con los hallazgos de anatomía patológica.

Establecer cuáles son los hallazgos ecográficos que más se relaciona con resultados histológicos malignos.

Conocer la discordancia entre el sistema de categorización TIRADS con los hallazgos anátomo patológicos de las lesiones tiroideas punzadas mediante PAAF con guía ecográfica.

Determinar si existen lesiones categorizadas como benignas, que hayan resultado malignas en el estudio de Anatomía Patológica (Falsos Negativos).

comprobar el VPP y VPN del equipo médico de dicha institución a la hora de clasificar un nódulo tiroideo según el sistema TIRADS.

\section{Material y métodos}

Tipo de investigación: Observacional descriptivo y retrospectivo.

Pacientes que asistieron al Servicio de Ecografía General del Centro Privado de Tomografía Computada Córdoba (Ciudad de Córdoba, Argentina) con indicación de punción-biopsia con aguja fina, guiada por ecografía (PAAF) de nódulo tiroideo, previamente diagnosticado $(n=81)$, en un periodo comprendido entre los meses de abril del año 2019, al mes de enero del año 2020 inclusive. El estudio fue aprobado por el comité de ética de nuestra institución, y todos los pacientes fueron correctamente informados sobre el procedimiento, firmando el Consentimiento Informado (anexo 1). Los instrumentos utilizados para la toma de muestras de los nódulos tiroideos no varían según el tipo de lesión. El método por el cual se realiza la 
punción es siempre guiado por ecografía en tiempo real (Figura 4).
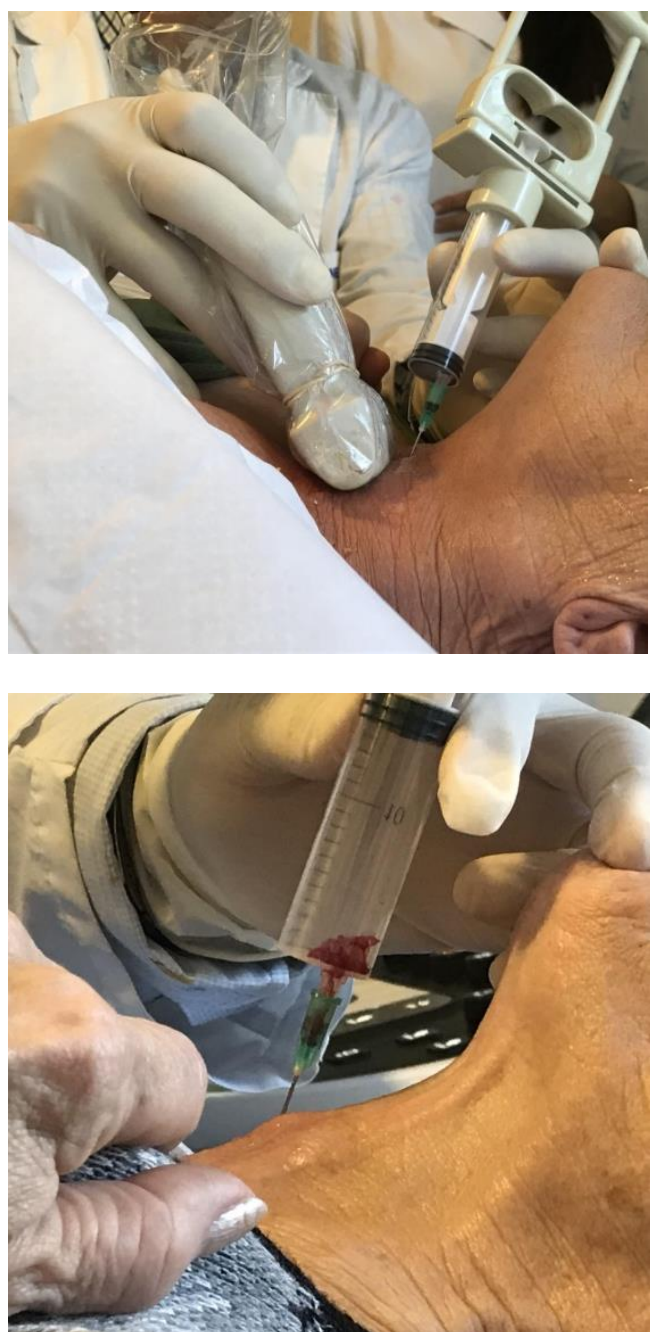

Figura 4. PAAF guiada por ecografía en tiempo real.

-Ecógrafo General Electric Logic S8 con transductores lineales de $8.5 \mathrm{MHz}$ para la realización de la elastografía.

-Ecógrafo Philips Affinity 70 con transductores lineales de $7.5 \mathrm{MHz}$ para guiar la punción.

-Cubre-transductor estéril.

-Portaobjetos.

- Aguja IM de $21 \mathrm{G}$.

- Jeringa de $20 \mathrm{ml}$.

- Aspirador montado a la jeringa.

Se realizó en un primer tiempo ecografía en modo $B$, Doppler color y elastografía SWE con medición de la ratio elastográfico, obteniendo valores en $\mathrm{kPa}$, en una sola toma correctamente obtenida.

En un segundo tiempo se procedió a realizar la punción aspirativa con aguja fina guiada (PAAF) por ecografía en tiempo real, con el paciente en decúbito dorsal, con el cuello en extensión, previas medidas de antisepsia cutánea. Se punzó con aguja $21 \mathrm{G}$, y jeringa de $20 \mathrm{ml}$ montada al aspirador.
Una vez tomadas las muestras, se coloca una gota del material extraído en el portaobjeto y es fijada en alcohol etílico al $70 \%$, el resto del material permanece en la jeringa para formarse un coágulo sólido, y son enviadas al laboratorio de Anatomía Patológica de nuestro centro para ser analizadas y categorizadas utilizando la clasificación Bethesda.

\section{Consideraciones éticas}

Todos los pacientes se realizaron por indicación de su médico de cabecera la PAAF.

Todos los pacientes firmaron previamente un consentimiento informado, donde quedan asentados los eventuales riesgos del procedimiento, y que los resultados podrían ser publicados, siendo sus datos personales de carácter confidencial.

Los datos personales fueron protegidos de acuerdo con la Ley de Protección de los Datos Personales (25.326), en sus Artículos $8^{\circ}, 9^{\circ}$ y $10^{\circ}$.

\section{Análisis estadístico}

Al obtener el resultado histológico, el mismo se cargó en una planilla de Microsoft Excel junto con las características ecográficas y el valor del ratio elastográfico $(\mathrm{kPa})$ del nódulo tiroideo, para realizar la correlación (anexo 2), la que posteriormente se utilizó para los procesamientos estadísticos. Para las variables cuantitativas se calcularon medidas de centralización y dispersión (media y desvío estándar), y para las variables categóricas se calcularon las distribuciones absolutas y porcentuales. Se aplicó el test de chicuadrado en la correlación de variables categóricas, con un nivel de significancia igual a 0,05. Además, se realizaron los cálculos de especificidad y sensibilidad, valores predictivos positivos (VPP) y valores predictivos negativos (VPN) para el score TIRADS. El software utilizado fue el Info Stat (v.2020).

\section{Resultados}

Se analizaron $\mathrm{n}=81$ pacientes de ambos sexos, mayores de 18 años, que presentaban lesión nodular sólida en glándula tiroides.

De las 81 PAAF realizadas, se obtuvieron 56 $(69,1 \%)$ con resultado anatomopatológico benigno (Bethesda II), $4(4,9 \%)$ con resultado borderline (Bethesda III), y 21 pacientes $(25,9 \%)$ con resultado maligno (Bethesda IV-V).

Los resultados de la ecografía previa a la punción del nódulo mostraron que la ecogenicidad de estos fue: hipoecogénica en el $38.3 \%$ de los pacientes, mixta en el $30,9 \%$, isoecogénica en el $29,6 \%$, en tanto que un porcentaje menor $(1,2 \%)$ fue hiperecogénica. 
Con respecto a la clasificación TIRADS, un 35,8\% de los pacientes de esta muestra fueron clasificados con valores de 4 a 5 (Figura 5). Un $51,9 \%$ fue de tipo 3 y un $12,3 \%$ de tipo 2 .

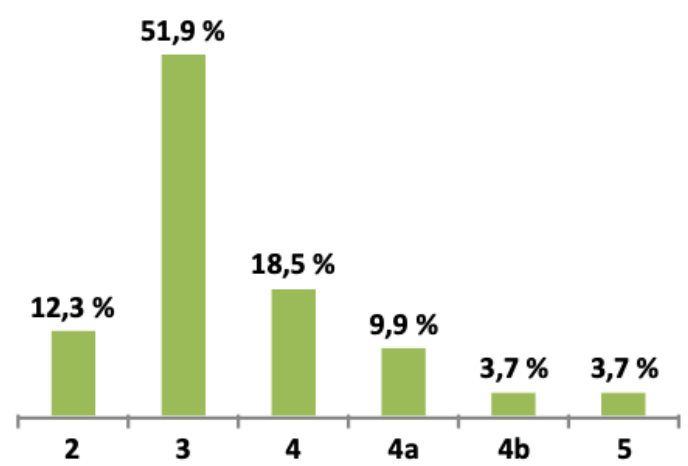

Figura 5. Distribución de pacientes según TIRADS $(\mathrm{n}=81)$.

\section{Resultados de la anatomía patológica}

En los casos con resultados benignos, las diferentes estirpes de nódulos fueron diagnosticados como nódulos benignos, bocio coloide e hiperplasia nodular (Tabla 3). También hubo casos de bocio coloide y bocio quístico. Entre los malignos se encontraron carcinoma papilar, folicular y folicular de células de Hürthle.

Tabla 3. Resultados histopatológicos

\begin{tabular}{|c|c|c|c|}
\hline Reporte & Anatomía Patológica & Cantidad & Porcentaje \\
\hline \multirow{7}{*}{$\begin{array}{l}\text { Benigno } \\
\text { (56 pacientes) }\end{array}$} & Nódulo benigno & 27 & $33,3 \%$ \\
\hline & Bocio coloide/hiperplasia nodular & 18 & $22,2 \%$ \\
\hline & Bocio coloide & 7 & $8,6 \%$ \\
\hline & Bocio quístico & 1 & $1,2 \%$ \\
\hline & Hiperplasia nodular & 1 & $1,2 \%$ \\
\hline & Nódulo/quiste coloide & 1 & $1,2 \%$ \\
\hline & Quiste coloide & 1 & $1,2 \%$ \\
\hline \multirow{3}{*}{$\begin{array}{l}\text { Maligno } \\
\text { (21 pacientes) }\end{array}$} & Carcinoma papilar & 14 & $17,3 \%$ \\
\hline & Carcinoma folicular & 5 & $6,2 \%$ \\
\hline & Carcinoma folicular de células de Hürthle & 2 & $2,5 \%$ \\
\hline $\begin{array}{l}\text { Dudoso } \\
\text { (4 pacientes) }\end{array}$ & AUS FLUS & 4 & $4.9 \%$ \\
\hline Total & & 81 & $100 \%$ \\
\hline
\end{tabular}

El porcentaje de malignidad en toda la muestra fue de $25,9 \%$, dentro de las categorías TI-RADS los reportes de malignidad fueron de menor a mayor: del 10\% en los pacientes con TIRADS 2 al 66,7\% para los pacientes con TIRADS 5. Al aplicar el test estadístico dio por resultado un valor de $p$ estadísticamente significativo ( $\mathrm{p}=0,0013)$.

El 12\% de los TIRADS 2 a 3 tuvieron un resultado patológico negativo (maligno) y en el caso de los TIRADS de 4 a 5 , este porcentaje se elevó a $52 \%$
(Tabla 4), la diferencia entre estas proporciones fue estadísticamente significativa $(\mathrm{p}<0,05)$.

Tabla 4. Sensibilidad y Especificidad

\begin{tabular}{|l|l|l|l|l|}
\hline TIRADS & Cantidad & Benignos & Malignos & p-valor \\
\hline 2 a 3 & 52 & $46(88 \%)$ & $6(12 \%)$ & \multirow{2}{*}{0,0001} \\
\hline 4 a 5 & 29 & $14(48 \%)$ & $15(52 \%)$ & \\
\hline
\end{tabular}

El $75,3 \%$ de todos los pacientes estuvo correctamente diagnosticado al correlacionar la cate-gorización TIRADS realizada por médicos de nuestro centro con los resultados de la anato-mía patológica, por lo que la sensibilidad del diagnóstico resultó de 71,4\% y la especificidad igual a $76,7 \%$. Además, el valor predictivo positivo (VPP) fue igual a $51,7 \%$ mientras que el valor predictivo negativo (VPN) fue de $88,5 \%$.

\section{Correlación entre Elastografía y Anatomía Patológica}

Utilizando un valor de corte de $65 \mathrm{kPa}$ (un valor menor es indicativo de benignidad y un va-lor mayor de malignidad), el 82,7\% de los casos (67 pacientes) se comprobó una correlación entre los valores de la Elastografía y la anatomía patológica (Figura 6) tanto resultados benig-nos como malignos, y en el $17,3 \%$ no lo hubo correlación (Figura 7, Tabla 5-6).

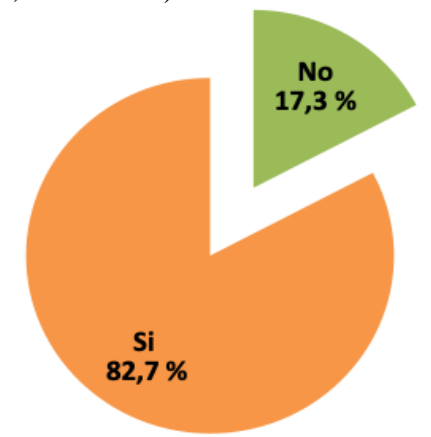

Figura 6. Correlación Elastografía / Anatomía Patológica $(n=81)$.

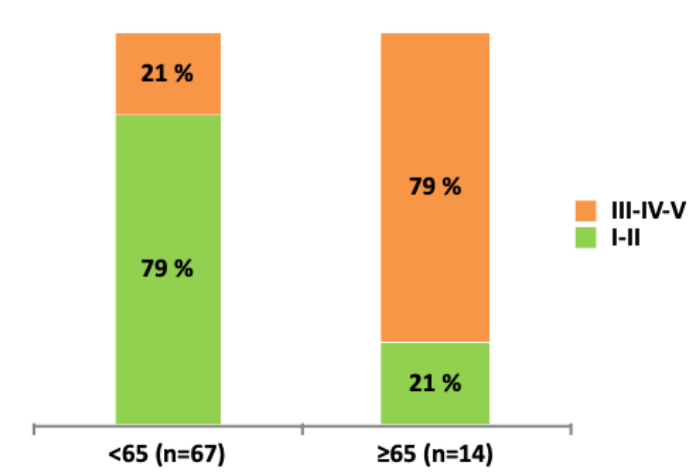

Figura 7. Distribución de la clasificación Bethesda según Elastografía $\mathrm{kPa}(\mathrm{p}=0,0001)$. 
Tabla 5. Sensibilidad y Especificidad (Elastografía Anatomía Patológica) Tabla 7. Sensibilidad y Especificidad (Elastografía - Anatomía Patológica)

\begin{tabular}{|l|l|l|}
\hline & Valor & Intervalo de confianza al 95\% \\
\hline Prevalencia de la enfermedad & $25,9 \%$ & $17 \%-37 \%$ \\
Pacientes correctamente diagnosticados & $79,0 \%$ & $68 \%-87 \%$ \\
Sensibilidad & $42,9 \%$ & $23 \%-66 \%$ \\
Especificidad & $91,7 \%$ & $81 \%-97 \%$ \\
VPP & $64,3 \%$ & $36 \%-98 \%$ \\
VPN & $82,1 \%$ & $70 \%-90 \%$ \\
\hline
\end{tabular}

Tabla 6. Distribución de las variables Calcificación, Elastografía y Ecogenicidad de nódulos tiroideos de pacientes con Bethesda IV-V $(n=21)$

\begin{tabular}{|c|c|c|c|}
\hline Variables & Categorías & Cantidad & Porcentaje \\
\hline \multirow{3}{*}{ Calcificación } & Macro & 3 & $14,2 \%$ \\
\hline & Micro & 7 & $33,3 \%$ \\
\hline & No & 11 & $52,3 \%$ \\
\hline \multirow{2}{*}{ Elastografia $\mathrm{kPa}$} & $<65$ & 10 & $47,6 \%$ \\
\hline & $\geq 65$ & 11 & $52,3 \%$ \\
\hline \multirow{2}{*}{ Ecogenicidad } & Isoecogénica & 7 & $33,3 \%$ \\
\hline & Hipoecogénica & 14 & $66,6 \%$ \\
\hline
\end{tabular}

\section{Discusión}

La ecografía tiroidea es el método de primera elección para la detección y caracterización de nódulos tiroideos, siendo la PAAF el método Gold standard para la detección del cáncer de tiroides. El Thyroid Imaging Reporting and Data System (TIRADS de Horvath et al. 2009), permite categorizar los nódulos tiroideos, para unificar terminología y seleccionar qué pacientes tienen indicaciones de PAAF. Esta clasificación propone 10 patrones ecográficos, y cada uno de ellos se asocia a un riesgo de cáncer. En 2011 G. Russ presenta la versión francesa del TI-RADS la cual incluye 6 categorías. En el mismo año, Kwak añade la categoría 4c a la versión de Horvath, no considerando el criterio de vascularización. Posteriormente, en el año 2013 G. Russ, simplifica esta categorización a 5 grupos. En 2017, la ACR (American College of Radiology) acordó una clasificación en la que se asignan puntajes según las características eco-gráficas del nódulo, para crear un léxico común, definir a qué nódulos realizar PAAF, y desarrollar directrices en el manejo de estos.

Diversos estudios demuestran que el rendimiento diagnóstico de la propia elastografía, o una combinación de elastografía y ecografía debe ser superior a la de la ecografía sola, ya que presenta un mayor VPN, no obstante, se necesitan más estudios prospectivos para mejorar su capacidad de diagnóstico.

El principal uso de esta innovadora herramienta es predecir la naturaleza maligna de un nódulo, para evitar realizar punciones innecesarias a nódulos benignos. Otro, es utilizarla para localizar y decidir qué nódulos son más susceptibles a la malignidad y guiar la elección de cuál es candidato a una PAAF.

Según el presente estudio, la prevalencia de cáncer de tiroides en la población estudiada es del 25,9\%. Los nódulos con resultado histológico maligno presentaron una predominancia de los siguientes hallazgos ecográficos: ecogenicidad mayormente hipoecogénica; sin calcificaciones, en segundo lugar, microcalcificaciones; y una rigidez $\geq 65 \mathrm{kPa}$ en la elastografía SWE.

En cuanto a la correlación anatomoelastográfica (Bethesda-kPa), se obtuvo una correlación del $83 \%$, una sensibilidad del $42,9 \%$ y una especificidad del $91,7 \%$, es decir que es más efectivo para determinar la ausencia de patología maligna; un valor predictivo positivo (VPP) del $64,3 \%$

y un valor predictivo negativo (VPN) del 82,1\%, menor que los estudios realizados por $\mathrm{Ab}$ delrahman et al. 21 y Kura et $\mathrm{al}^{22}$.

Lin y cols ${ }^{23}$, señalaron que la SWE era una técnica de gran valor para descartar la presencia de cáncer de tiroides debido al alto VPN, de modo que aconsejaban incluirla en los protocolos de estudio de nódulos tiroides. Sin embargo, reconocen que las conclusiones de su trabajo deben tomarse con cautela, puesto que la prevalencia de cáncer era sustancialmente más alta que en la población general, puesto que se trataba de pacientes ya seleccionados que iban a ser sometidos a biopsia.

\section{Conclusión}

La elastografía es una técnica ya desarrollada en algunos órganos como la mama y el hígado. Para la glándula tiroidea hay varios estudios, a pesar de que fueron realizados con muestras pequeñas, presentaron resultados prometedores, demostrando un gran potencial como nueva herramienta para el diagnóstico de cáncer de tiroides, en una población con alta prevalencia de nódulos tiroideos, donde la mayoría de estos resultan histológicamente benignos.

Además, es una buena herramienta de diagnóstico complementario a la ecografía convencional y al TIRADS, es decir, no sustituye a la escala de grises, pero aumenta la sensibilidad $y$ especificidad, y así, permite disminuir el número de punciones innecesarias o mejorar la elección del 
nódulo candidato a la PAAF en pacientes que presentan múltiples nódulos, limitando los gastos y disminuyendo la potencial morbilidad de la PAAF y la cirugía.

Cabe resaltar, que, si bien aún no hay consensos sobre los valores de corte elastográficos para clasificar los hallazgos benignos y malignos, por lo pronto cada institución debería establecer sus propios valores de corte según basados es estudios poblacionales y el equipamiento utilizado.

\section{Bibliografía}

1. Niepomniszce H, Sala M, Danilowicz K, Pitoia F, et al. Epidemiology of palpable goiter in greater buenos aires in an iodinesufficient area. Medicina (B Aires), 2004;64(2):142.

2. Urciuoli C, Abelleira E, Balonga M, Arevalo de Cross G, et al. Prevalencia de enfermedades tiroideas en una población del área metropolitana de Buenos Aires. Revista Argentina de Endocrinología y Metabolismo 2006; 53:67-72.

3. Surraco M, Marquez M, Mereshian P, Geres $A$ et al. Campaña de detección de enfermedades tiroideas en la ciudad de Córdoba. Hospital San Roque, Hospital Nacional de Clínicas, Hospital Córdoba, Hospital Misericordia, Fundación para el progreso de la medicina, Laboratorio Hospital Córdoba, FAMAF-CIEM.UNC.

4. Guth S, Theune U, Aberle J, Galach A, et al. Very high prevalence of thyroid nodules detected by high frequency (13 MHz) ultrasound examination. European Journal Clinical Investigation 2009;39(8):699-706.

5. Tan GH, Gharib H. Thyroid incidentalomas: Management approaches to nonpalpable nodules discovered incidentally on thyroid imaging. Ann Intern Med 1997;126: 226-231.

6. Nishiyama R, Ludwig G, Thompson N. The prevalence of small papillary thyroid carcinoma in 100 consecutive necropsies in an American population. Radiation-Associated Thyroid Carcinoma. New York: Grune \& Stratton 1977;123-35.

7. Thyroid Imaging Reporting \& Data System (TI-RADS ${ }^{\mathrm{TM}}$ ), American College of Radiology.

8. Pinto Blazquez J, del Valle Manteca A, Solera Arroyo JC, Cuesta Martínez L, et al. Sistema Bethesda en el diagnóstico citopatológico de la patología de tiroides. Otorrinolaringológica de Castilla y León, Cantabria y La Rioja. 2014;5(8):66-74.

9. Sigrist RMS, Liau J, Kaffas AE, Chammas MC and Willmann JK. Ultrasound Elastography: Review of Techniques and Clinical Applications. Theranostics. 2017; 7:1303-1329.

10. Garra BS. Elastography: history, principles, and technique comparison. AbdomImaging. 2015; 40:680-697.

11. Asensio del Barrio $\mathrm{C}$ Efectividad diagnóstica y seguridad de distintas modalidades de elastografía en indicaciones oncológicas. Una revisión de revisiones sistemáticas y metaanálisis. Red Española de Agencias de Evaluación de Tecnologías y Prestaciones del SNS. Agencia de Evaluación de Tecnologías Sanitarias (AETS) - Instituto de Salud Carlos III, Ministerio de Ciencia, Innovación y Universidades. Madrid. 2018. Informes de Evaluación de Tecnologías Sanitarias.

12. Friedrich-Rust M, Romenski O, Meyer G, et al. Acoustic Radiation Force Impulse Imaging for the evaluation of the thyroid gland: a limited patient feasibility study. Ultrasonics 2012; 52:69-74.

13. Veyrieres JB, Albarel F, Lombard JV, et al. A threshold value in Shear Wave elastography to rule out malignant thyroid nodules: a reality? European Journal of Radiology 2012; 81:3965-72.

14. Gu J, Du L, Bai M, et al. Preliminary study on the diagnostic value of acoustic radiation force impulse technology for differentiating between benign and malignant thyroid nodules. Journal of Ultrasound in Medicine 2012;31(5):763-771.

15. Bojunga J, Dauth N, Berner C, et al. Acoustic radiation force impulse imaging for differentiation of thyroid nodules. PLoS ONE 2012;7(8): e42735.

16. Hou XJ, Sun AX, Zhou XL, et al. The application of Virtual Touch tissue quantification (VTQ) in diagnosis of thyroid lesions: A preliminary study. European Journal of Radiology 2013; 82:797-801.

17. Sebag F, Vaillant-Lombard, Berbis J, et al. Shear wave elastography: a new ultrasound imaging mode for the differential diagnosis of benign and malignant thyroid nodules. Journal of Clinical Endocrinology and Metabolism 2010;95(12):5281-8. 
18. Sporea I, Vlad M, Bota S, et al. Thyroid stiffness assessment by acoustic radiation force impulse elastography (ARFI). Ultraschall in der Medizin 2011; 32:281-5.

19. Sporea I, Sirli R, Bota S, Vlad M, et al. ARFI elastography for the evaluation of diffuse thyroid gland pathology: preliminary results. World Journal of Radiology 2012; 4:174-8.

20. Magri F, Chytiris S, Capelli V, et al. Shear wave elastography in the diagnosis of thyroid nodules: feasibility in the case of coexistent chronic autoimmune Hashimoto's thyroiditis. Clinical Endocrinology

(Oxf) 2012;76(1):137-41.
21. Abdelrahman SF, Ali FH, El-Sayed Khalil M, El Masry MR. Ultrasound elastography in the diagnostic evaluation of indeterminate thyroid nodules. Egypti J Radiol Nuclear Med. 2015;46(3):639-48.

22. Kura M, Ballarino C, Tamagnone F, Campagno B, et al. Relación entre el valor del ratio elastográfico y la clasificación citológica de Bethesda en la patología tiroidea. Revista Argentina de Radiología 2014; 78:128-137.

23. Lin $\mathrm{P}$, Chen $\mathrm{M}$, Liu B, Wang $\mathrm{S}$ Et al. Diagnostic performance of shear wave elastography in the identification of malignant thyroid nodules: a meta-analysis. European Radiology 2014; 24:2729-2738.

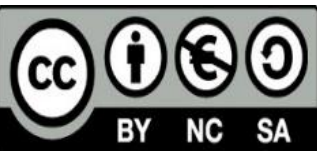

